\title{
Robolution: Real Time Predictive Analytics for Industrial Robots
}

\author{
Sajal Suhane, Pramod D. Patil, Ravi Mishra, Simran Koul, Ridima Shukla, Jyoti Rao
}

\begin{abstract}
Robots have been playing a very important role in our day-to-day lives and will be a necessity in the coming future. Whenever we hear automation, the first thing that strikes our mind is a robot performing the given task. But if a robot fails to do the task, it could cost an individual or corporate a huge financial loss. In this study, we have learned the working of various robots and drawbacks that hold them back. For this work, we did make a study of drives used in the robot and after that applied the machine learning algorithms to predict the classification of whether the robot will function properly or not, based on the data of drive(s).
\end{abstract}

Keywords: Machine Learning, Data Science, Robotics

\section{INTRODUCTION}

Robots now-a-days have witnessed a large number of users since the process of automation hasbegun. Today, we need Robots in every field, right from agriculture to performing various tasks in corporates. 'Robolution' is the term that is used for this era of Robots. But the question arises do we know will the robot perform the given task accurately or even if it does it accurately, does it do it the right way. To overcome such difficulties and ease the human decision making for assigning task to robot, we need algorithms to predict the next result of robot[1], using its dynamic dataset which is integrated with the cloud[2]. The program will be connected to cloud system and will provide output with a prediction graph.

The objective of this workis predictive maintenance of industrial robots and the possibility of building a condition monitoring system based on the data analysis of the robot's locomotion which brings the work of individuals at one place.The aim of this study is also to detect the robot's accuracy for performing a task correctly.This can be checked by observing a robot for a certain period and then using that dataset for prediction[3].

\section{Revised Manuscript Received on February 05, 2020.}

* Correspondence Author

Sajal Suhane*, Department of Computer Engineering, Dr. D.Y. Patil Institute of Technology, Pimpri, Pune, India. Email: ssuhane31@gmail.com

Dr. Pramod D. Patil, Department of Computer Engineering, Dr. D.Y Patil Institute of Technology, Pimpri, Pune, India. Email: pdpatiljune@gmail.com

Ravi Mishra, Department of Computer Engineering, Dr. D.Y. Pati Institute of Technology, Pimpri, Pune, India. Email: mishra.ravi@mail.com

Simran Koul, Department of Computer Engineering, Dr. D.Y. Patil Institute of Technology, Pimpri, Pune, India. Email: simran.koul@mail.com

Ridima Shukla, Department of Computer Engineering, Dr. D.Y. Pati Institute of Technology, Pimpri, Pune, India. Email: ridimashuklawork@gmail.com

Dr. Jyoti Rao, Department of Computer Engineering, Dr. D.Y. Patil Institute of Technology, Pimpri, Pune, India. Email: jyotiasawale@gmail.com

(C) The Authors. Published by Blue Eyes Intelligence Engineering and Sciences Publication (BEIESP). This is an open access article under the CC BY-NC-ND license (http://creativecommons.org/licenses/by-nc-nd/4.0/)
Present work majorly focuses on Industrial Robots because their robots perform critical tasks and its failure could be a hefty cost for the industry. Analyzing robot behavior from prior data is the fundamental basis of a data science approach like machine learning.

\section{LITERATURE SURVEY}

"Data Analytics for Predictive Maintenance of Industrial Robots"(Borgi, Tawfik, et al.)presents the predictive maintenance of industrial machines based on the data analysis of robot's power measurements. A predictive modelling approach is proposed in the paper, to detect robot manipulator accuracy errors based on robot's current data analysis for predictive maintenance purposes. Also, an experimental procedure is carried out to oversee the correlation between the robot accuracy error and a set of extracted features from current time-series, and to evaluate the proposed predictive modelling.

"Big Data Analytics for Real Time Systems" (Dutta, K., \& Jayapal, M.) discusses the use of Big Data and its impact in our day to day lives. It focuses on the fact that Data, if processed at the right time could provide us with some eyeopening insights. An overview of the Big Data Analytics for Real Time Systems and focus on its challenges (3V's of data), research trends and accuracy of results has been studied in the paper.

"An Empirical Comparison of Supervised Learning Algorithms" (Caruana, Rich, and Alexandru NiculescuMizil)comes up with an approach to find the best suited algorithms for Big Data problems using supervised learning methods: SVMs, neural nets, logistic regression, naive bayes, memory-based learning, random forests, decision trees, bagged trees, boosted trees, and boosted stumps. It also examines the effectof calibrating the models via Platt Scaling and Isotonic Regression has on their performance.

"KnowRob-knowledge processing for autonomous personal robots" (Tenorth, Moritz, and Michael Beetz) portrays how data could be processed in the robots in order to get the most out of robot. Knowledge extraction from the given data without hampering the working of robot is one of the major methods discussed in the paper. A Knowledge processing framework and its implementation has been explained which works in almost all the robots (as concluded in the paper).

Since, most of the work is done in focused areas such as Robotics, Real time data processing, comparison of algorithms etc. This study tends to bring forward the work done in an interdisciplinary manner for ease of human. Therefore, the work proposes an addition to the existing technology for better robot efficiency and working. 


\section{Robolution: Real Time Predictive Analytics for Industrial Robots}

\section{PROPOSED SYSTEM}

\section{A. System Overview}

A robot has a controller and drives attached to it which concurrently executes command(s) given by its administrator. Functioning of every drive(s) and storing the data generated by drive(s) to cloud is handled by the controller. Various machine learning algorithms are implemented on the motherboard of controller and whenever drive generates new data, the previous data is fetched from the cloud and model(s) are trained accordingly to classify new data. Different algorithms run on the controller simultaneously in a passive manner (without harming the current actions of robot), and the algorithm with best performance metrics is considered for the prediction. The prediction of various drive(s) of robot is sent to the administrator through a service installed on the linked remote device via Wi-Fi locally [4][5].

Now, with the help of this prediction the human administrator can know which drive will work properly and which will fail in the next action, and thus industry can take necessary actions in order to minimize the costs of failure.

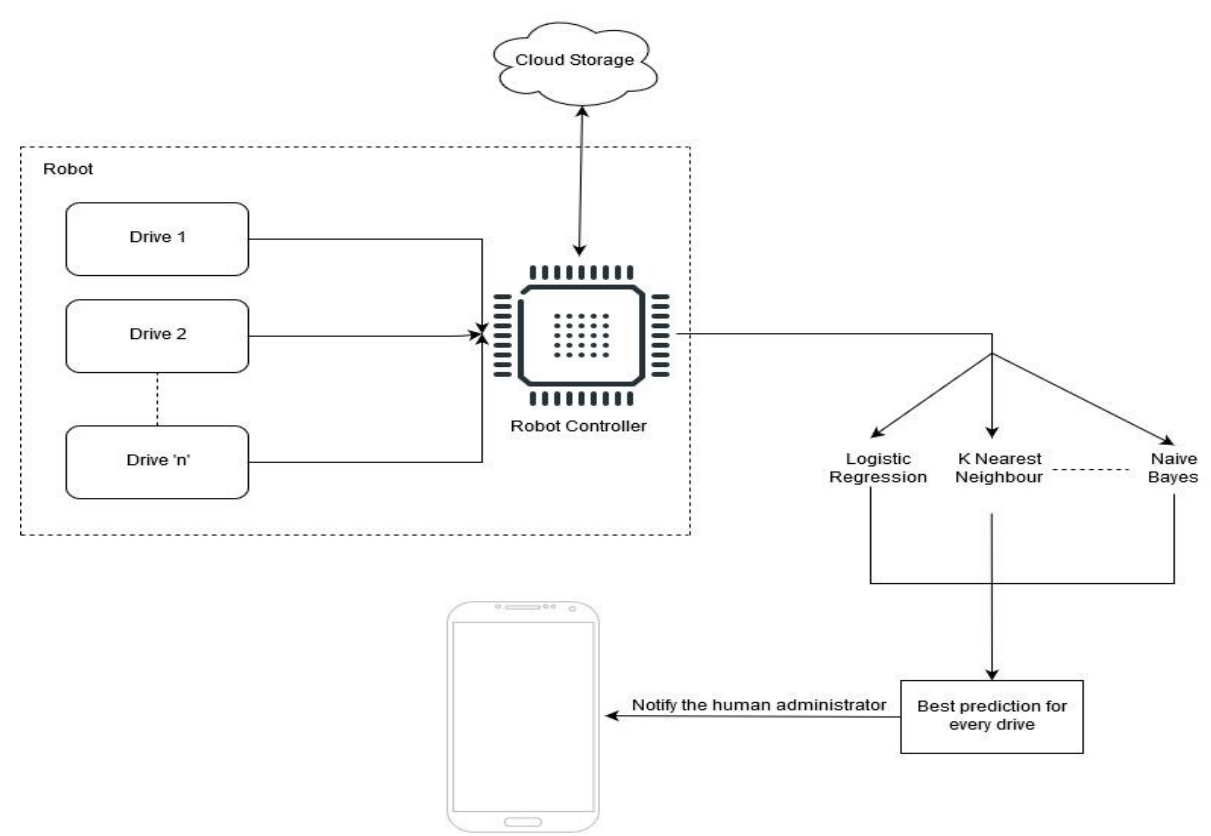

Fig-I: Architecture Diagram for the proposed model

\section{A. Data Sets}

The data generated by drives is mostly in .csv format. Data generally varies from robot to robot based on its use and implementation. A typical drive database consists various features such as time, positions, external torques, commanded pose and end effectors [6][7][8]. A total of around 2,10,000 tuples training data was used. A sample of dataset has been shown below:

Table-I: Sample dataset of robot with their features

\begin{tabular}{|c|c|c|c|c|c|}
\hline Timestamp & position & external_torque & commanded_pose & end_effectors & work_done \\
\hline $\mathbf{1 2} / \mathbf{1 6} / \mathbf{2 0 1 8}$ & 5.1 & 3.5 & 1.4 & 0.2 & 1 \\
\hline $\mathbf{1 2 / 1 7} / \mathbf{2 0 1 8}$ & 4.9 & 3 & 1.4 & 0.2 & 0 \\
\hline $\mathbf{1 2 / 1 8} / \mathbf{2 0 1 8}$ & 4.7 & 3.2 & 1.3 & 0.2 & 0 \\
\hline $\mathbf{1 2 / 1 9} / \mathbf{2 0 1 8}$ & 4.6 & 3.1 & 1.5 & 0.2 & 0 \\
\hline $\mathbf{1 2} / \mathbf{2 0} / \mathbf{2 0 1 8}$ & 5 & 3.6 & 1.4 & 0.2 & 1 \\
\hline $\mathbf{1 2} / \mathbf{2 1} / \mathbf{2 0 1 8}$ & 5.4 & 3.9 & 1.7 & 0.4 & 1 \\
\hline $\mathbf{1 2} / \mathbf{2 2} / \mathbf{2 0 1 8}$ & 4.6 & 3.4 & 1.4 & 0.3 & 1 \\
\hline $\mathbf{1 2} / \mathbf{2 3} / \mathbf{2 0 1 8}$ & 5 & 3.4 & 1.5 & 0.2 & 1 \\
\hline $\mathbf{1 2} / \mathbf{2 4} / \mathbf{2 0 1 8}$ & 4.4 & 2.9 & 1.4 & 0.2 & 0 \\
\hline $\mathbf{1 2} / \mathbf{2 5} / \mathbf{2 0 1 8}$ & 4.9 & 3.1 & 1.5 & 0.1 & 0 \\
\hline $\mathbf{1 2} / \mathbf{2 6} / \mathbf{2 0 1 8}$ & 5.4 & 3.7 & 1.5 & 0.2 & 0 \\
\hline $\mathbf{1 2} / \mathbf{2 7} / \mathbf{2 0 1 8}$ & 4.8 & 3.4 & 1.6 & 0.2 & 0 \\
\hline
\end{tabular}




\begin{tabular}{|c|c|c|c|c|c|}
\hline $\mathbf{1 2 / 2 8 / 2 0 1 8}$ & 4.8 & 3 & 1.4 & 0.1 & 0 \\
\hline $\mathbf{1 2 / 2 9 / 2 0 1 8}$ & 4.3 & 3 & 1.1 & 0.1 & 0 \\
\hline $\mathbf{1 2 / 3 0 / 2 0 1 8}$ & 5.8 & 4 & 1.2 & 0.2 & 1 \\
\hline $\mathbf{1 2 / 3 1 / 2 0 1 8}$ & 5.7 & 4.4 & 1.5 & 0.4 & 1 \\
\hline $\mathbf{1 / 1 / 2 0 1 9}$ & 5.4 & 3.9 & 1.3 & 0.4 & 1 \\
\hline $\mathbf{1 / 2 / 2 0 1 9}$ & 5.1 & 3.5 & 1.4 & 0.3 & 0 \\
\hline $\mathbf{1 / 3 / 2 0 1 9}$ & 5.7 & 3.8 & 1.7 & 0.3 & 1 \\
\hline
\end{tabular}

B. Performance Results

Various predictive algorithms such as Naïve Bayes, K Nearest Neighbors, Logistic Regression, Random Forest, Support Vector Machines, Bagging and Boosting were used on the robot dataset to evaluate which algorithm works best.
The parameters used for evaluating a model are Accuracy, Standard Deviation, Total time of running, time taken by model for training according to data and the scoring time. A summarized comparison has been shown below:

Table- II: Tabular comparision of various models

\begin{tabular}{|c|c|c|c|c|c|}
\hline Model & Accuracy & $\begin{array}{c}\text { Standard } \\
\text { Deviation }\end{array}$ & Total Time & Training Time & $\begin{array}{c}\text { Scoring } \\
\text { Time }\end{array}$ \\
\hline Naïve Bayes & $94.4 \%$ & $\pm 2.4 \%$ & $664 \mathrm{~ms}$ & $4 \mathrm{~ms}$ & $103 \mathrm{~ms}$ \\
\hline K Nearest Neighbor & $97.2 \%$ & $\pm 1.1 \%$ & $2 \mathrm{~s}$ & $1 \mathrm{~ms}$ & $\sim 0 \mathrm{~ms}$ \\
\hline Logistic Regression & $95.1 \%$ & $\pm 1.8 \%$ & $3 \mathrm{~s}$ & $2 \mathrm{~ms}$ & $1 \mathrm{~ms}$ \\
\hline $\begin{array}{c}\text { Support Vector } \\
\text { Machine }\end{array}$ & $79.2 \%$ & $\pm 4.1 \%$ & $12 \mathrm{~s}$ & $2 \mathrm{~s}$ & $103 \mathrm{~ms}$ \\
\hline $\begin{array}{c}\text { Random Forest } \\
\text { Bagging }\end{array}$ & $77.9 \%$ & $\pm 3.9 \%$ & $7 \mathrm{~s}$ & $4 \mathrm{~s}$ & $915 \mathrm{~ms}$ \\
\hline Boosting & $91.2 \%$ & $\pm 2.3 \%$ & $985 \mathrm{~ms}$ & $70 \mathrm{~ms}$ & $1 \mathrm{~ms}$ \\
\hline
\end{tabular}

\section{Comparision based on Robot's Data}

$120.00 \%$

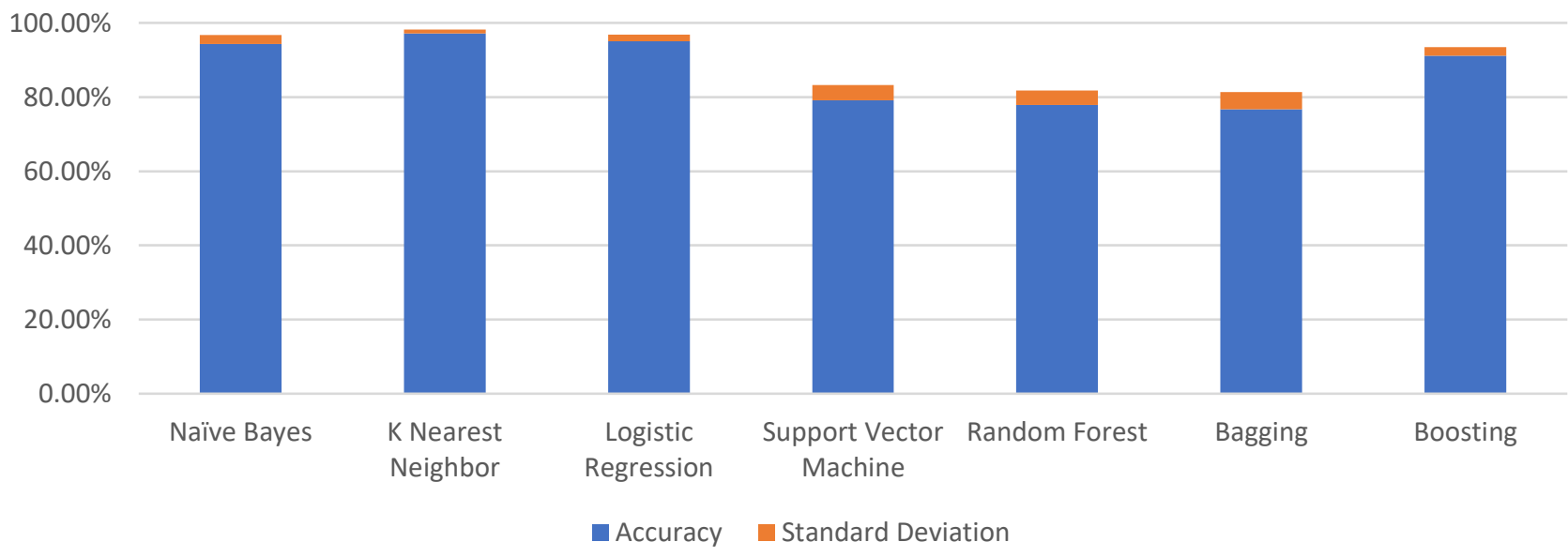

Fig - II: Graphical performance comparison between various predictive algorithms

Published By:

Blue Eyes Intelligence Engineering

925 \& Sciences Publication 


\section{RESULTS AND DISCUSSION}

From study, it has been observed that K Nearest Neighbor, Logistic Regression, and Naïve Bayes shows exceptional accuracy of $97.2 \%, 95.1 \%$, and $94.4 \%$ respectively. Naïve Bayes algorithm has the fastest execution time of $664 \mathrm{~ms}$ in total and a standard deviation of $\pm 2.4 \%$.

Robot's dataset is unpredictable. The research work done till now explains how to deal with outliers and missing values. But the integration of those methods with robot's drive dataset and using that dataset in models to predict robots' action has not been done which has been discussed in this paper. The data changes with working of drive(s) of robot and might have null values and missing values. The models have been built such that they remove noise from the data and then process it to get higher accuracy values with greater precision. Amongst various other models, Naïve Bayes performs exceptionally and is robust with almost all types of robots.

The system is based on drive predictions on the features and data generated by each drive of the robot as an input to the system.

\section{CONCLUSION AND FUTURE SCOPE}

The field of Automation and Machine Learning has made substantial progress in the last decade. Learning methods such as boosting, random forests, bagging, and SVMs achieve excellent performance that would have been difficult to obtain just 20 years ago.

In the present work, a survey of existing robot drive systems was conducted and the drawbacks of each system were studied. The present work suggests additional functionality to the existing system. Furthermore, it tries to leverage the functionalities of the system by selecting features with the best correlation to get more accurate results in optimal time. The system is based on drive predictions on the features and data generated by each drive of the robot as an input to the system. The system could use Reinforcement Learning and calculate best path the robot should follow in order to get maximum rewards. It will allow the robot to execute certain tasks with the utmost efficiency. Integration with voice home automation systems could be an effective addition to the system for ease of the end-user.

With present work, it is revealed, Logistic Regression, K Nearest Neighbors, and Naïve Bayes have the best performance among all the and accuracy values with the robot datasets.

\section{ACKNOWLEDGMENT}

This research was fully supported by our guide and mentor Dr. Pramod D. Patil, Principal and Professor, Dr. D.Y. Patil Institute of Technology, Pimpri, Pune-18. I'm thankful to Brabo Robotics and Automation Ltd. who provided insight and expertise that greatly assisted the research.

\section{REFERENCES}

1. Borgi, Tawfik, et al. "Data analytics for predictive maintenance of industrial robots." 2017 International Conference on Advanced Systems and Electric Technologies (IC_ASET). IEEE, 2017.

2. Dutta, Kamalika, and Manasi Jayapal. "Big data analytics for real time systems." Big Data analytics seminar. 2015.

3. Caruana, Rich, and Alexandru Niculescu-Mizil. "An empirical comparison of supervised learning algorithms." Proceedings of the 23rd international conference on Machine learning. ACM, 2006.
4. Tenorth, Moritz, and Michael Beetz. "KnowRob-knowledge processing for autonomous personal robots." 2009 IEEE/RSJ International Conference on Intelligent Robots and Systems. IEEE, 2009.

5. Lee, Jay, et al. "Industrial big data analytics and cyber-physical systems for future maintenance \& service innovation." Procedia Cirp 38 (2015): 3-7.

6. Bauer, Eric, and Ron Kohavi. "An empirical comparison of voting classification algorithms: Bagging, boosting, and variants." Machine learning 36.1-2 (1999): 105-139.

7. Blake, C., \& Merz, C. (1998). UCI repository of machine learning databases.

8. Marz, Nathan, and James Warren. Big Data: Principles and best practices of scalable realtime data systems. O'Reilly Media, 2013.

\section{AUTHORS PROFILE}

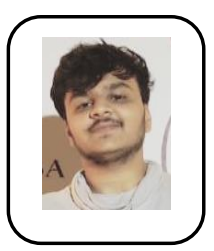

Sajal Suhane, is an undergraduate student of Computer Engineering at Dr. D.Y. Patil Institute of Technology, Pimpri, Pune. He has pursued various courses on Data Science and possess a keen interest in this field. He is a member and student branch coordinator of professional body Computer Society of India (CSI).

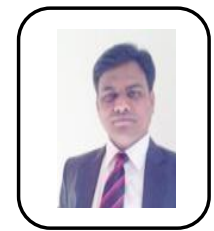

Dr. Pramod D. Patil, an alumnus of college of engineering. (COEP) Pune, holds Masters in Computer Engineering and Ph.D. from COEP. He has total 19 years of experience in Academics, Research and Industry. He has 40 plus research articles in National \& International Journals and Conferences to his credit. He is currently principal of DIT Pimpri, Pune and is member of professional society such as CSI, ACM, IEEE, and ISTE. He is reviewer of many peer reviewed international journals like ACM, Springer and Elsevier.

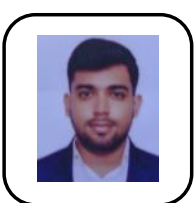

Ravi Mishra, is an undergraduate student of Compute Engineering at Dr. D.Y. Patil Institute of Technology, Pimpri, Pune. He has pursued various courses on Java and its applications and has a keen interest in this field.He is a member of professional body Computer Society of India (CSI).

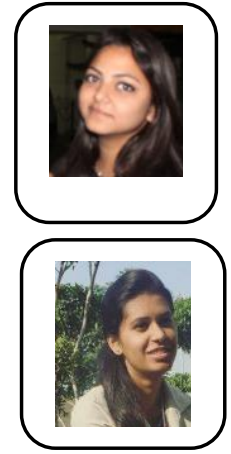

Simran Koul, is an undergraduate student of Computer Engineering at Dr. D.Y. Patil Institute of Technology, Pimpri, Pune. She is inclined towards database structures and its connectivity. Along with it she is proficient in Java and its applications. She is a member of professional body Computer Society of India (CSI).

Ridima Shukla, is an undergraduate student of Computer Engineering at Dr. D.Y. Patil Institute of Technology, Pimpri, Pune. She is inclined towards database structures and its connectivity. Along with it she is proficient in Java and its applications. She is a member of professional body Computer Society of India (CSI).

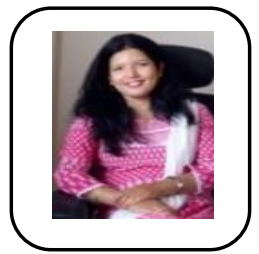

Dr. Jyoti Raohas, total 18 years of Teaching ExperienceinComputerEngineering.Sheisworking as Professor in D.Y. Patil Institute of Technology, Pimpri. She is approved Post Graduate TeacheratSPPU.ShecompletedherPhDin2016on topic Novel and efficient Visual Cryptographyschemeforprivacyprotection. She has 2 Patent Published in IPR. She has more than 20 papers published in international journal. 